\title{
A calibration method of multiple rangefinders system
}

\author{
Hideto Kameshima, Yukio Sato, Hideo Saito \\ Keio University, 3-14-1 Hiyoshi, Kouhoku-ku, Yokohama-shi Kanagawa-ken, Japan, 223-8522
}

\begin{abstract}
The calibration method for a multiple rangefinders system is proposed in this paper. Generally, multiple rangefinders are required to obtain the whole surface shape of a large object such as a human body. The proposed method solves range data registration by a prior calibration using a reference plane with rectangular markers. The world coordinates system is defined on the reference plane. Because range data have about two hundred thousands of 3-D points, the normal vector of the reference plane is accurately estimated by fitting the regression plane to the 3-D points. If the Z-axis of the world coordinates system for our calibration method is defined as the axis which cross meets the reference plane, it is determined by the normal vector. On the other hand the $\mathrm{X}$ and $\mathrm{Y}$ axes are defined as the horizontal line and vertical line of rectangular markers. They are determined by detecting and extracting the rectangular markers from the intensity image. Therefore, the orientation of each rangefinder is estimated based on the world coordinates system. In the experiments, the multiple rangefinders system which consists of twelve rangefinders is used. Experimental results indicate that the RMSE is $2.3 \mathrm{~mm}$ in the case of measuring a cylinder object.
\end{abstract}

Keywords: Rangefinder, 3-D Measurement, Multiple Measurement, Calibration

\section{INTRODUCTION}

An active rangefinder is able to acquire an accurate object's surface shape. Some rangefinders can obtain a color intensity image with range data speedily. At the moment, the principal tools for sizing up a human body are still a ruler and a tape measure. However, a rangefinder will become a practical tool for measuring human body shape, because we can measure not only a chest and abdominal circumferences but also the area and the volume of a human body.

Generally, a rangefinder acquires only the part of the object's surface shape. Because the measurable space of a rangefinder is limited, multiple rangefinders are required to obtain the whole surface shape of a large object such as a human body. In these circumstances, range data acquired from several viewpoints need to be precisely registered to obtain a whole surface shape. If the range data are not precisely registered, it causes gaps among the range data and makes object's circumferences incorrect. For this reason, range data registration is one of the important problems for 3-D measurement by multiple rangefinders system.

Regarding a registration problem, there are two major approaches. One is the approach which calibrates the geometric relation among rangefinders using a reference object beforehand [1]. If rangefinders are calibrated by this method once, each range data is registered at the same time as measuring it. The other is the approach which registers and integrates the range data by software processing after measuring. This is the technique for matching the overlapped parts among the range data called ICP [2,3]. Even if the geometric relation among rangefinders is not known, the range data is registered based on geometric features of each range data. However, if the range data has smooth surface without any hole, edge or corner, the range data is not always registered using ICP. In addition, if any range data does not have overlapped region, it is not always possible to solve the registration problem after measuring.

In this paper, we proposed a calibration method for the multiple rangefinders system which accurately integrates the multiple range data. In our study, this problem is solved by a prior calibration for multiple rangefinders using a reference plane with rectangular markers. Before measuring, the orientation of each rangefinder is estimated from its range data and color intensity images by temporarily measuring the reference plane. Because range data usually consist of hundred thousands of 3-D points, the normal vector of the reference plane is accurately estimated by fitting the regression plane to the 3-D points. If the Z-axis of the world coordinate system for our calibration method is defined as the axis which cross meets the reference plane, it is determined by the normal vector. On the other hand the $\mathrm{X}$ and $\mathrm{Y}$ axes of the world coordinate system are defined as the horizontal line and vertical line of rectangular markers on the reference plane. They are determined by detecting and extracting the rectangular markers from the intensity image acquired by the range data. Therefore, the orientation of each rangefinder is estimated based on the world coordinate system. Additionally, a method

Three-Dimensional Image Capture and Applications 2008, edited by Brian D. Corner, Masaaki Mochimaru, Robert Sitnik Proc. of SPIE-IS\&T Electronic Imaging, SPIE Vol. 6805, 68050P, @ 2008 SPIE-IS\&T · 0277-786X/08/\$18 


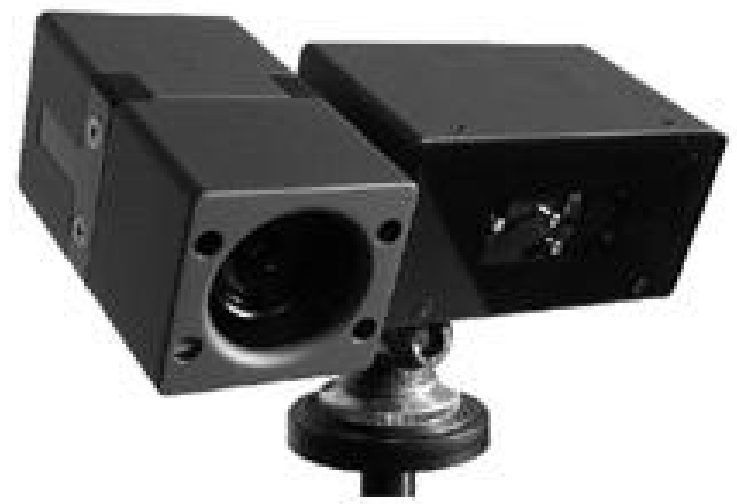

Fig. 1. Rangefinder

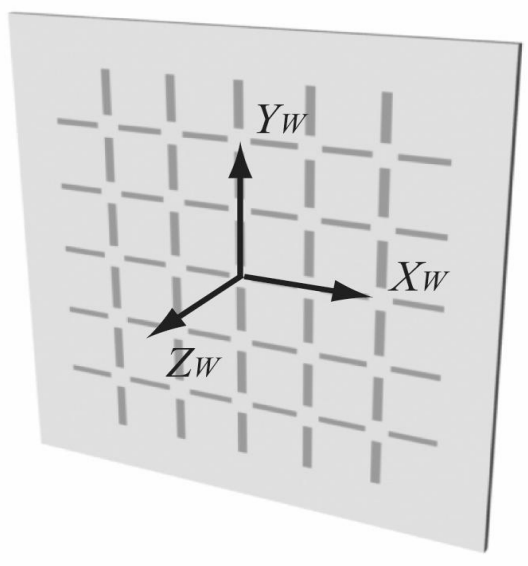

Fig. 2. Calibration plane

which corrects the geometric distortion of the range data is proposed. So that we would like to solve the calibration problem for large scale multiple rangefinder systems, the accuracy of the registration is influenced by the accuracy of each rangefinder. Hence, in some cases, the correcting method is needed for the accurately calibration.

In the experiments, the multiple rangefinder systems which consist of twelve rangefinders are used. Experimental results indicate that the RMSE is $2.3 \mathrm{~mm}$ in the case of measuring a cylinder object. In the case of human body measurement, there are no perceptible gaps among the range data of a man.

\section{CALIBRATION FOR MULTIPLE RANGEFINDER SYSTEMS}

\subsection{Compact Rangefinder}

To measure an object shape accurately and speedily, we use the compact rangefinder [4] as shown in Fig.1. This rangefinder is composed of a CCD camera ( $640 \mathrm{x} 480$ pixels) and a laser scanner. It is able to capture a range images within 0.5 seconds, due to the light pattern from the scanner is generated by improved space encoding method using pattern shifting $[5,6]$. When we measure a target placed one meter ahead, the measurement error is found to be $2 \mathrm{~mm}$.

\subsection{World coordinate system on calibration plane}

In the case of the calibration of 2-D multiple camera system which consists of two or more cameras, something for relating to the camera coordinate system to world coordinate system must be used. For example, a cubic object or a cylinder object, an object of which size and shape are known is usually used. One of the simplest objects for reference objects must be a plane with some geometric markers on it. In this case, the geometric relations are estimated to extract the markers on the reference plane from the captured image. To improve the accuracy of the camera calibration, the orientation or the position of markers must be extracted stably and precisely. Therefore, the accuracy of the camera calibration is basically depends on the limit of the image capturing. Especially, in the case of large multiple range finder systems, it is difficult to accurately calibrate the system from only captured intensity images. However, when we face the calibration problem of multiple rangefinders system, we can approach the calibration problem based on the quite different concept because of acquiring a range data.

A range map is a kind of 2-D image which has an absolute distance from a camera to the surface point. Our rangefinder is able to acquire not only range maps but also color intensity image. For instance, when the rangefinder observes measuring a plane object, a 3-D several hundred thousand points on the plane with spatial coordinates are obtained together with a surface texture. Each measurement point of the range map is stored based on the camera coordinate system. Therefore, once the regression plane is fitted to the measured 3-D points, the accuracy of the regression plane is expected to be high, and the normal vector of the plane is useful for one of the axis of the world coordinate system. 

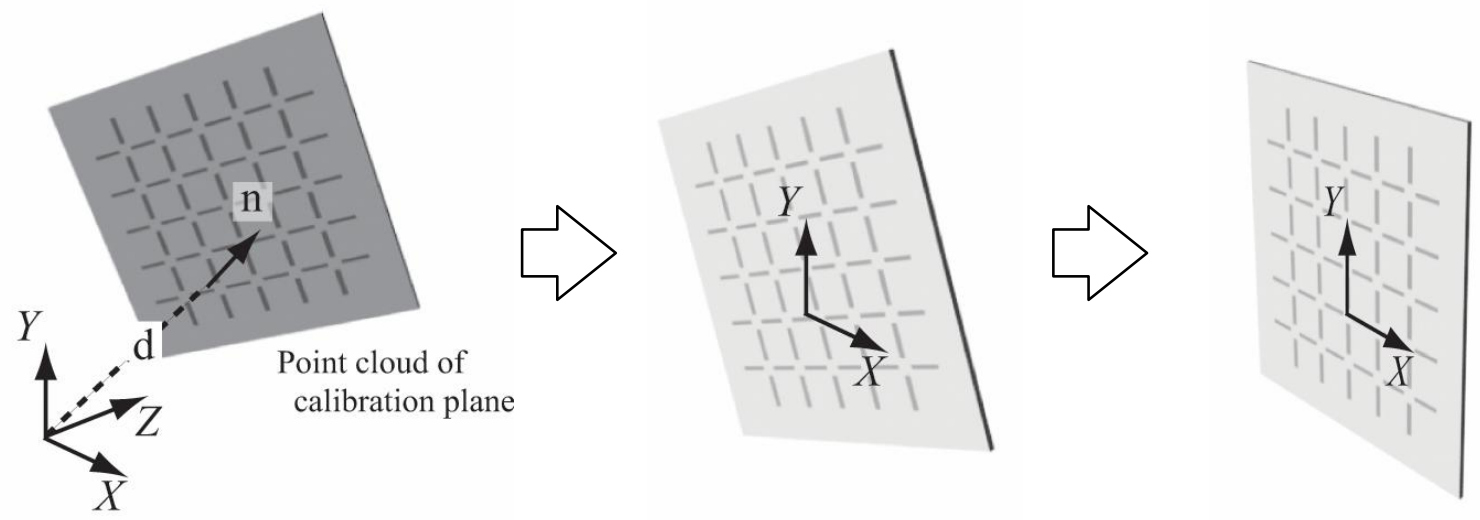

Fig. 3. Integration into the world coordinate system on the calibration plane

In our method, the world coordinate axis $Z_{W}$ is defined as the normal vector of the regression plane. Assuming that each range finder is possible to capture only the part of the calibration plane, it is difficult to determine the world coordinate system from only the geometrical shape of the plane. However, using the geometrical pattern which gives the orientation and the position from the world coordinate system on the calibration plane, the other axes $\left(X_{W}, Y_{W}\right)$ can be estimated from the pattern. Thus, the other axes of the world coordinate system are defined on the calibration plane (Fig.2). $X_{W}, Y_{W}$ are allocated as the horizontal direction and the vertical direction of the rectangular marker of the calibration plane respectively. The proposed method solves the calibration problem between the camera coordinate system and the world coordinate system using the range data and the intensity image which captures the part of the calibration plane (Fig. 3).

\subsection{Calibration}

In general, the range map is described as the camera coordinate system which depended on the camera position and direction to the world coordinate system. To obtain a whole shape of an object by multiple rangefinder systems, each range map is needed to be integrated in the world coordinate system. Thus, the transformation from the camera coordinate system to the world coordinate system is required for the rangefinder calibration, and three axes of the world coordinate system need to be extracted from the range map using the calibration plane.

The equation of a plane in the 3-D space is represented as

$$
a x+b y+c z+1=0
$$

These coefficients are accurately estimated by fitting the plane to 3-D points of the range map, and the normal vector $\mathbf{n}$ and the distance between the plane and the origin $d$ are given by

$$
\begin{aligned}
& \mathbf{n}=\frac{\left[\begin{array}{lll}
a & b & c
\end{array}\right]^{T}}{\sqrt{a^{2}+b^{2}+c^{2}}} \\
& d=\frac{1}{\sqrt{a^{2}+b^{2}+c^{2}}}
\end{aligned}
$$

We get the rotation matrix $\mathbf{R}_{1}$ and the translation vector $\mathbf{T}_{1}$ which adjust $\mathbf{n}$ to the Z-axis of the world coordinate system $\mathbf{e}_{z}$ 


$$
\begin{gathered}
\mathbf{R}_{1}=\mathbf{u} \mathbf{u}^{T}+\left(\mathbf{I}-\mathbf{u u}^{T}\right) \cos \theta+\left[\begin{array}{ccc}
0 & -u_{z} & u_{y} \\
u_{z} & 0 & -u_{x} \\
-u_{y} & u_{x} & 0
\end{array}\right] \sin \theta \\
\mathbf{T}_{1}=\left[\begin{array}{lll}
0 & 0 & d
\end{array}\right]^{T}
\end{gathered}
$$

where $\mathbf{u}$ is the axis of the rotation and $\theta$ is the angle given by

$$
\begin{gathered}
\mathbf{u}=\mathbf{n} \times \mathbf{e}_{z} \\
\theta=\cos ^{-1} \mathbf{n} \cdot \mathbf{e}_{z}
\end{gathered}
$$

A 3-D calibration point of $i$ line $j$ row from the range data and the corresponding point on the calibration plane are denoted by $\mathbf{c}_{i j}=\left[\begin{array}{lll}x_{i, j}^{c} & y_{i, j}^{c} & z_{i, j}^{c}\end{array}\right]^{T}$ and $\mathbf{w}_{i j}=\left[\begin{array}{lll}x_{i, j}^{w} & y_{i, j}^{w} & 0\end{array}\right]^{T}$ respectively. The Euclidean transform which adjust all of $\mathbf{c}_{i j}$ to $\mathbf{w}_{i j}$ in $X_{W}-Y_{W}$ is given by

$$
\left[\begin{array}{c}
x_{i, j}^{w} \\
y_{i, j}^{w}
\end{array}\right]=\left[\begin{array}{cccc}
\alpha & -\beta & 0 & t_{x} \\
\beta & \alpha & 0 & t_{y}
\end{array}\right]\left[\begin{array}{cc}
\mathbf{R}_{1} & \mathbf{T}_{1} \\
\mathbf{0} & 1
\end{array}\right]\left[\begin{array}{c}
x_{i, j}^{c} \\
y_{i, j}^{c} \\
z_{i, j}^{c} \\
1
\end{array}\right]
$$

Thus, let the rotation matrix $\mathbf{R}_{2}$ and the translation vector $\mathbf{T}_{2}$ be

$$
\begin{gathered}
\mathbf{R}_{2}=\left[\begin{array}{ccc}
\cos \phi & -\sin \phi & 0 \\
\sin \phi & \cos \phi & 0 \\
0 & 0 & 1
\end{array}\right] \quad \mathbf{T}_{2}=\left[\begin{array}{c}
t_{x} \\
t_{y} \\
0
\end{array}\right] \\
\phi=\tan ^{-1} \frac{\beta}{\alpha}
\end{gathered}
$$

Thus, the Euclidean transformation from the camera coordinate system to the world coordinate system is shown by the next expression.

$$
\left[\begin{array}{c}
x_{w} \\
y_{w} \\
z_{w} \\
1
\end{array}\right]=\left[\begin{array}{cc}
\mathbf{R}_{2} & \mathbf{T}_{2} \\
\mathbf{0} & 1
\end{array}\right]\left[\begin{array}{cc}
\mathbf{R}_{1} & \mathbf{T}_{1} \\
\mathbf{0} & 1
\end{array}\right]\left[\begin{array}{c}
x_{c} \\
y_{c} \\
z_{c} \\
1
\end{array}\right]
$$

\subsection{Experimental results}

The calibration for multiple rangefinder systems was done by using the proposal method. In the experiment, three measuring units (Fig.4 (a)), where three compact rangefinders are installed, were located in front of the calibration plane (Fig.4 (b)). The plastic board of $850 \mathrm{~mm}$ in width, $1800 \mathrm{~mm}$ in height and $7 \mathrm{~mm}$ in thickness was used as the calibration plane in this experiment. The pattern sheet with the rectangular pattern (30 rows and 9 columns) was stuck on the surface of the calibration plane and each size of rectangular pattern was $60 \mathrm{~mm}$. 


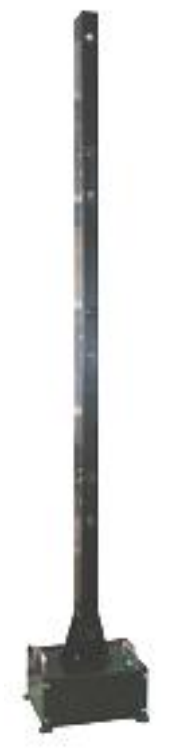

(a) Measurement unit

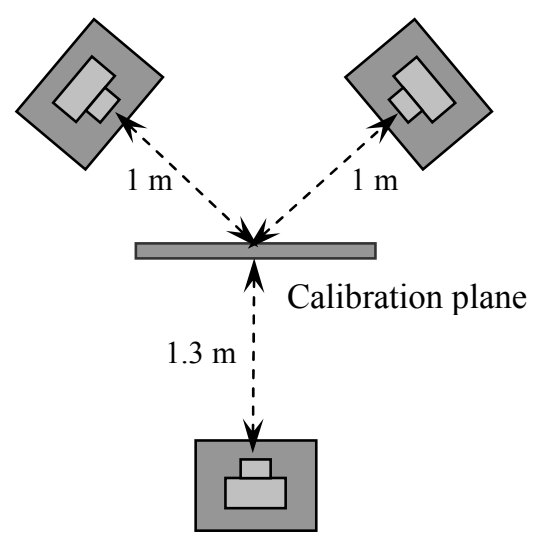

(b) Rangefinder configuration

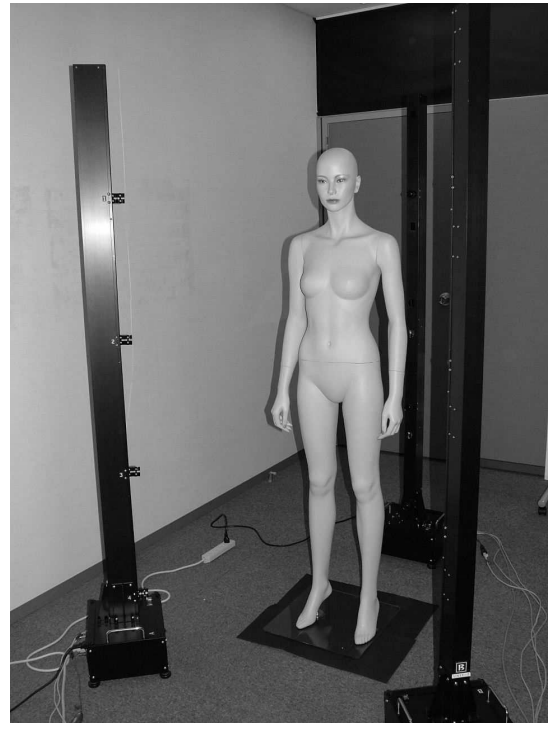

(c) Measurement system

Fig. 4. Rangefinder configuration of experiment

A cylinder object with $200 \mathrm{~mm}$ diameter and $2000 \mathrm{~mm}$ height was used for evaluating the calibration accuracy, and it was placed at the center of the measuring units. The 3-D point cloud of the cylinder object is shown in Fig. 5 (a) and its sectional image at $800 \mathrm{~mm}$ height is shown in Fig. 5 (b). In Fig. 5 (b), the solid line shows the fitted circle to the measurement data. The fitting error was found to be $6.4 \mathrm{~mm}$ RMSE which is defined as root mean square of the deference of the cylinder radius and the distance between a position of 3-D point and the center position of cylinder objects.

\section{CORRECTING DISTROTION}

\subsection{Correcting distortion}

Regarding with the calibration for multiple rangefinder systems, the error of registration is a large problem, because the length of the circumference and the volume are greatly influenced by the gap among the range data. The cause of the gaps is the calibration error of the intrinsic parameters of the rangefinder. However, it is difficult to estimate all of the intrinsic rangefinder parameters exactly in practice. Thus, correcting distortion of the range data is tried in our research. The linear correction matrix $\mathbf{K}$ is defined as

$$
\mathbf{K}=\left[\begin{array}{cccc}
k_{11} & k_{12} & k_{13} & k_{14} \\
k_{21} & k_{22} & k_{23} & k_{24} \\
k_{31} & k_{32} & k_{33} & k_{34} \\
0 & 0 & 0 & 1
\end{array}\right]
$$

When the calibration plane is measured, the Euclidean transform $\left(\mathbf{R}_{1}, \mathbf{R}_{2}\right.$ and $\left.\mathbf{T}_{1}, \mathbf{T}_{2}\right)$ is estimated from the range data. If the calibration plane in various postures is measured several times, $\mathbf{K}$ is solved from the calibration points of the camera coordinate system and the world coordinate system by the linear least square method. 


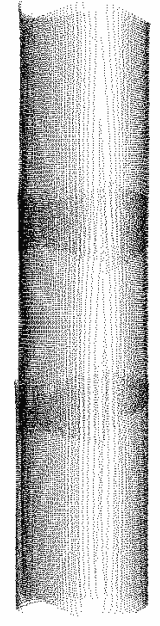

(a) 3-D points

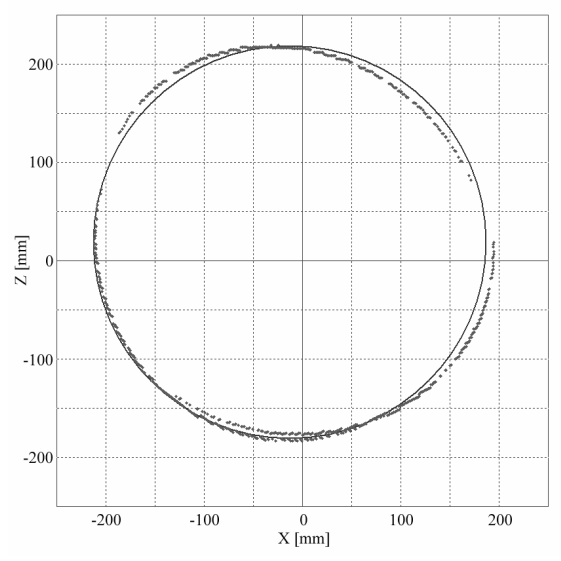

(b) Sectional image at $800 \mathrm{~mm}$ heigh

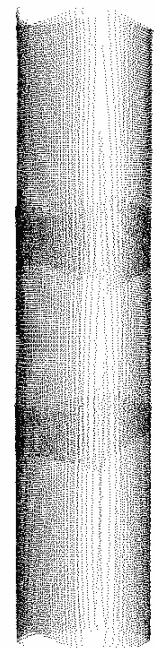

(a) 3-D points

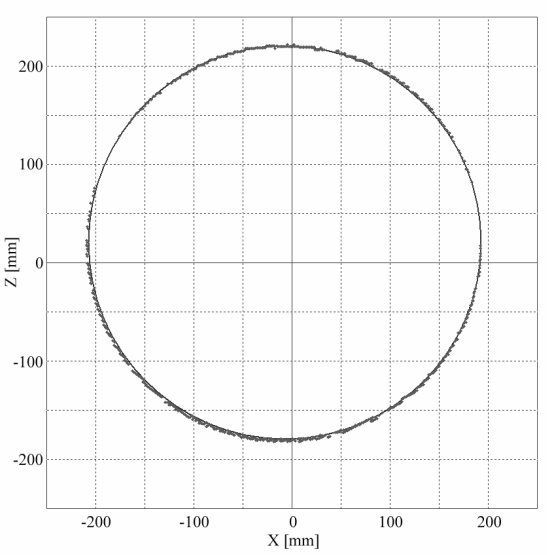

(b) Sectional image at $800 \mathrm{~mm}$ heigh1

Fig. 5. Measurement result of the cylinder

Fig. 6. Measurement result of the cylinder with distortion correction

$$
\left[\begin{array}{cc}
\mathbf{R}_{1} & \mathbf{T}_{1} \\
\mathbf{0} & 1
\end{array}\right]^{-1}\left[\begin{array}{cc}
\mathbf{R}_{2} & \mathbf{T}_{2} \\
\mathbf{0} & 1
\end{array}\right]^{-1}\left[\begin{array}{c}
x_{i, j}^{w} \\
y_{i, j}^{w} \\
0 \\
1
\end{array}\right]=\left[\begin{array}{cccc}
k_{11} & k_{12} & k_{13} & k_{14} \\
k_{21} & k_{22} & k_{23} & k_{24} \\
k_{31} & k_{32} & k_{33} & k_{34} \\
0 & 0 & 0 & 1
\end{array}\right]\left[\begin{array}{c}
x_{i, j}^{c} \\
y_{i, j}^{c} \\
z_{i, j}^{c} \\
1
\end{array}\right]
$$

where $\mathbf{K}$ is the correction matrix which reduces the error of the calibration points in the camera coordinate system.

\subsection{Experimental results}

To evaluate the accuracy for this method, the cylinder object was measured in the same situation to 3.3. In this experiment, we have measured the calibration plane five times while rotating the plane by about 15 degrees. After calibrating four rangefinders, the geometric distortion of each range data are corrected and integrated into 3-D point cloud. Fig. 6 (a) shows 3-D point cloud corrected by the proposal method, and Fig. 6 (b) is its sectional image at $800 \mathrm{~mm}$ height. Compared with Fig. 5 (b), the points of the range map is on the solid line of the fitted circle in Fig. 6 (b). In this case, the RMSE of the fitting error is found to be $3 \mathrm{~mm}$ and it is about $0.3 \%$ of the distance from the rangefinder to the cylinder object. Thus, the integration error for this method is enough small as long as the range data are corrected.

\section{CONCLUSION}

In this paper, we proposed a calibration method for the multiple rangefinders system which accurately integrates the multiple range data. To calibrate extrinsic parameters of each rangefinder, we use a calibration plane with rectangular markers as the reference object. The world coordinate system is defined on the calibration plane, and the position and the orientation of each rangefinder is able to be estimated based on the range map of the calibration plane measured by each rangefinder. As the experiment result indicates, nine range maps of the cylinder object are accurately integrated without any gaps by the proposed method. 

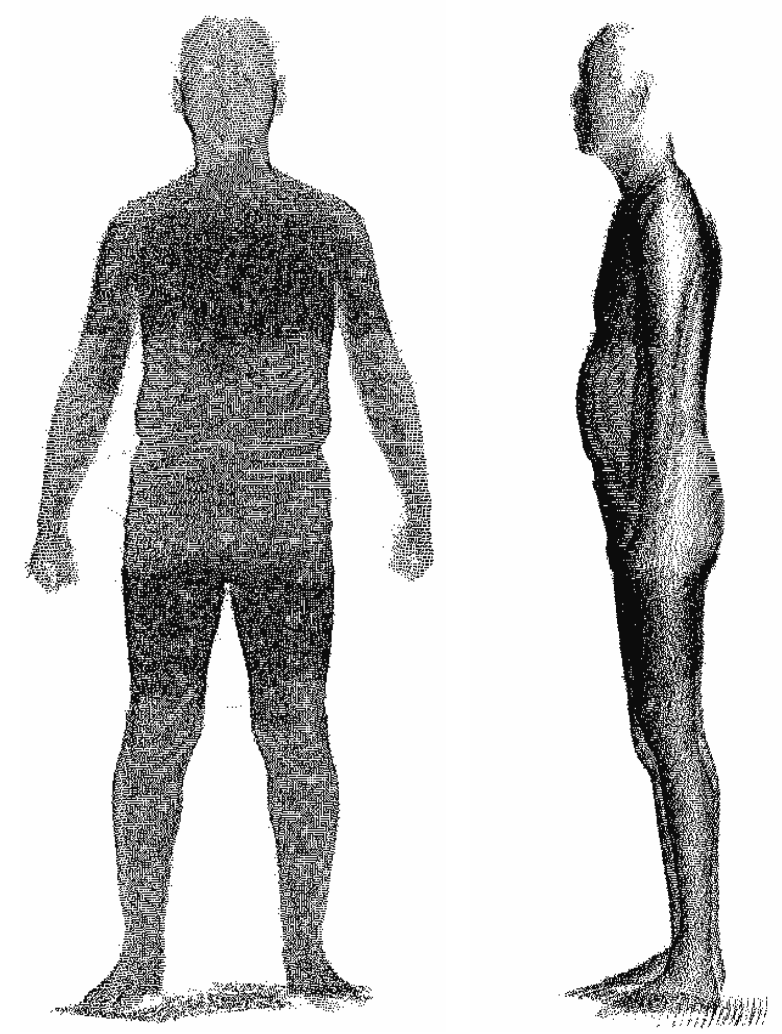

Fig. 7. Measurement result of human body

The field of Humanmetrics is need of a 3-D measurement system which accurately obtains a human body shape, such as a face, arms and legs. Because nobody can keep a pose for a long time, one of the most important things of measuring a human body is a measurement time, and the multiple rangefinder systems is useful. The multiple rangefinder systems called Cartesia 3-D HUMAN BODY SCANNER (SPACEVISON Inc.) is used for measuring a large object such as a human body. This system is consisted of twelve rangefinders and can acquire twelve range data and intensity images. Each rangefinder captures a range image within 0.5 seconds, the total measurement time of this system is about 3 seconds as long as a couple of rangefinders capture range images simultaneously. In Fig. 7, the measurement data for a man where integrated from nine range maps by the proposal method. As a result, there are no perceptible gaps among the range data.

\section{ACKNOWLEDGEMENTS}

The work presented in this paper is mainly supported by CREST, JST (Research Area: Foundation of technology supporting the creation of digital media contents).

\section{REFERENCES}

1 Koichiro Yamauchi, Yukio Sato, "3D Human Body Measurement by Multiple Range Images," icpr, pp. 833-836, 18th International Conference on Pattern Recognition (ICPR'06) Volume 4, 2006 
P.J.Besl and N.D.McKay, "A Method for Registration of 3-D Shapes", IEEE Trans. Pattern Anal. Machine Intell, Vol.14, No.2, pp.239-256, Feb. 1992.

3 Y.Chen, G.Medioni, "Object modeling by registration of multiple range images", Image and Vision Computing, vol.10, no.3, April 1992.

4 http://www.space-vision.jp/

$5 \quad$ Y.Sato and M.Otsuki, “Three-Dimensional Shape, Reconstruction by Active Rangefinder," In IEEE Conf. Computer Vision and Pattern Recognition, pp.142-147, 1993.

6 K.Hattori and Y.Sato, "Accurate Rangefinder with Laser Pattern Shifting," Proc. International Conference on Pattern Recognition, Vol.3, pp.849-853, 1996. 